\title{
Impact of diabetes on colorectal cancer stage and mortality risk: a population-based cohort study
}

\author{
Judy K. Qiang ${ }^{1} \cdot$ Rinku Sutradhar ${ }^{2,3} \cdot$ Vasily Giannakeas $^{3,4} \cdot$ Dominika Bhatia $^{2} \cdot$ Simron Singh $^{3}$. \\ Lorraine L. Lipscombe ${ }^{1,2,3,4}$
}

Received: 18 September 2019 / Accepted: 17 December 2019 / Published online: 28 January 2020

(C) Springer-Verlag GmbH Germany, part of Springer Nature 2020

\begin{abstract}
Aims/hypothesis Diabetes is associated with an increased incidence of colorectal cancer (CRC). There exists conflicting evidence regarding the impact of diabetes on CRC-specific mortality (herein also referred to as cancer-specific mortality). The objectives of this study were to determine whether diabetes is associated with a more advanced CRC stage at diagnosis and with higher all-cause and cancer-specific mortality.

Methods This retrospective cohort study used linked, population-based health databases from Ontario, Canada. Among individuals diagnosed with CRC from 2007 to 2015, we compared the likelihood of presenting with later- (III or IV) vs early- (I or II) stage CRC between patients with and without diabetes adjusting for relevant covariates. We then determined the association between diabetes and all-cause and CRC-specific mortality, after adjusting for CRC stage at diagnosis and other covariates.

Results Of the 44,178 individuals with CRC, 11,822 (26.7\%) had diabetes. After adjustment for CRC screening and other covariates, individuals with diabetes were not more likely to present with later-stage CRC (adjusted OR 0.97, 95\% CI 0.93, 1.01). Over a median follow-up of 2.63 (interquartile range [IQR] 0.97-5.10) years, diabetes was associated with higher all-cause mortality (adjusted HR 1.08, 95\% CI 1.04, 1.12) but similar cancer-specific survival (adjusted HR 1.0, 95\% CI 0.95, 1.06).

Conclusions/interpretation Individuals with diabetes who develop CRC are not more likely to present with a later stage of CRC and have similar cancer-specific mortality compared with those without diabetes. Diabetes was associated with higher all-cause mortality in CRC patients, indicating that greater attention to non-cancer care is needed for CRC survivors with diabetes.
\end{abstract}

Keywords Colorectal cancer mortality $\cdot$ Colorectal cancer stage $\cdot$ Diabetes mellitus

\author{
Abbreviations \\ $\mathrm{CCO} \quad$ Cancer Care Ontario \\ $\mathrm{CHF} \quad$ Congestive heart failure \\ CRC Colorectal cancer
}

Electronic supplementary material The online version of this article (https://doi.org/10.1007/s00125-020-05094-8) contains peer-reviewed but unedited supplementary material, which is available to authorised users.

Lorraine L. Lipscombe

Lorraine.Lipscombe@wchospital.ca

1 Department of Medicine, Division of Endocrinology and Metabolism, University of Toronto, Toronto, ON, Canada

2 Institute of Health Policy, Management and Evaluation, University of Toronto, Toronto, ON, Canada

3 ICES, Toronto, ON, Canada

4 Women's College Research Institute, Women's College Hospital, 76 Grenville Street, Room 6424, Toronto, ON M5S 1B2, Canada

$\begin{array}{ll}\text { FOBT } & \text { Faecal occult blood test } \\ \text { GI } & \text { Gastrointestinal } \\ \text { IQR } & \text { Interquartile range } \\ \text { MI } & \text { Myocardial infarction } \\ \text { MOHLTC } & \text { Ministry of Health and Long-Term Care } \\ \text { OCR } & \text { Ontario Cancer Registry } \\ \text { OHIP } & \text { Ontario Health Insurance Plan } \\ \text { ORG } & \text { Office of the Registrar General }\end{array}$

\section{Introduction}

Diabetes is associated with an increased incidence of several cancers, particularly malignancies of the colorectum, endometrium and breast [1-7]. Colorectal cancer (CRC) is the third most commonly diagnosed cancer, representing over $6 \%$ of all cancers globally [8]. While the association between diabetes and cancer has been partly attributed to detection bias [1, 9], a 


\section{Research in context}

\section{What is already known about this subject?}

- Individuals with diabetes mellitus have an increased rate of colorectal cancer (CRC)

- Previous investigations on the association between diabetes and survival in individuals with CRC have yielded mixed results

\section{What is the key question?}

- Do individuals with diabetes present with a later stage at CRC diagnosis and have higher cancer-specific and overall mortality risk compared with those without diabetes?

\section{What are the new findings?}

- After adjustment for appropriate covariates, individuals with diabetes are as likely to present with a later stage of CRC as those without diabetes

- Individuals with diabetes have higher all-cause mortality but similar CRC-specific mortality compared with those without diabetes

\section{How might this impact on clinical practice in the foreseeable future?}

- Diabetes has a greater impact on non-cancer than cancer mortality risk for individuals with CRC, indicating that greater attention to non-cancer care is needed for those with diabetes and CRC

recent European cohort study found that individuals with diabetes had an increased risk of gastrointestinal cancers compared with controls even after applying a 1 year lag period to adjust for detection bias [10]. The presence of insulin resistance and compensatory hyperinsulinaemia has been proposed as a mechanism of increased cancer risk, through the tumour growthpromoting effects of insulin and IGF-1 [11].

Diabetes has also been associated with increased all-cause mortality after cancer diagnosis, with the strongest relationship seen for colorectal, breast and endometrial cancers [12]. However, the extent to which diabetes affects cancer vs noncancer mortality risk is uncertain, particularly for CRC. Some studies have shown decreased cancer-specific survival for diabetes patients with CRC [13, 14], whereas two studies found that diabetes status was not associated with a worse CRC-specific mortality $[15,16]$. These conflicting results may be a reflection of methodological variations, differences in populations studied and heterogeneity in diabetes care, as well as in geographical locations in which previous studies were conducted.

One of the most important prognostic factors in CRC is stage at diagnosis [17]. CRC may be curable in stages I, II and III, with 10 year CRC-specific survival rates estimated at $89 \%, 80 \%$ and $63 \%$, respectively [18]. Stage IV CRC is not curable, with a significantly poorer 10 year survival rate of approximately $10 \%[18,19]$. Evidence suggests that overall cancer screening rates may be lower among individuals with diabetes, which may lead to later cancer detection and more advanced stage at diagnosis [20]. For example, we and others have shown that women with diabetes have lower breast and cervical cancer screening rates [21-24], and more advanced-

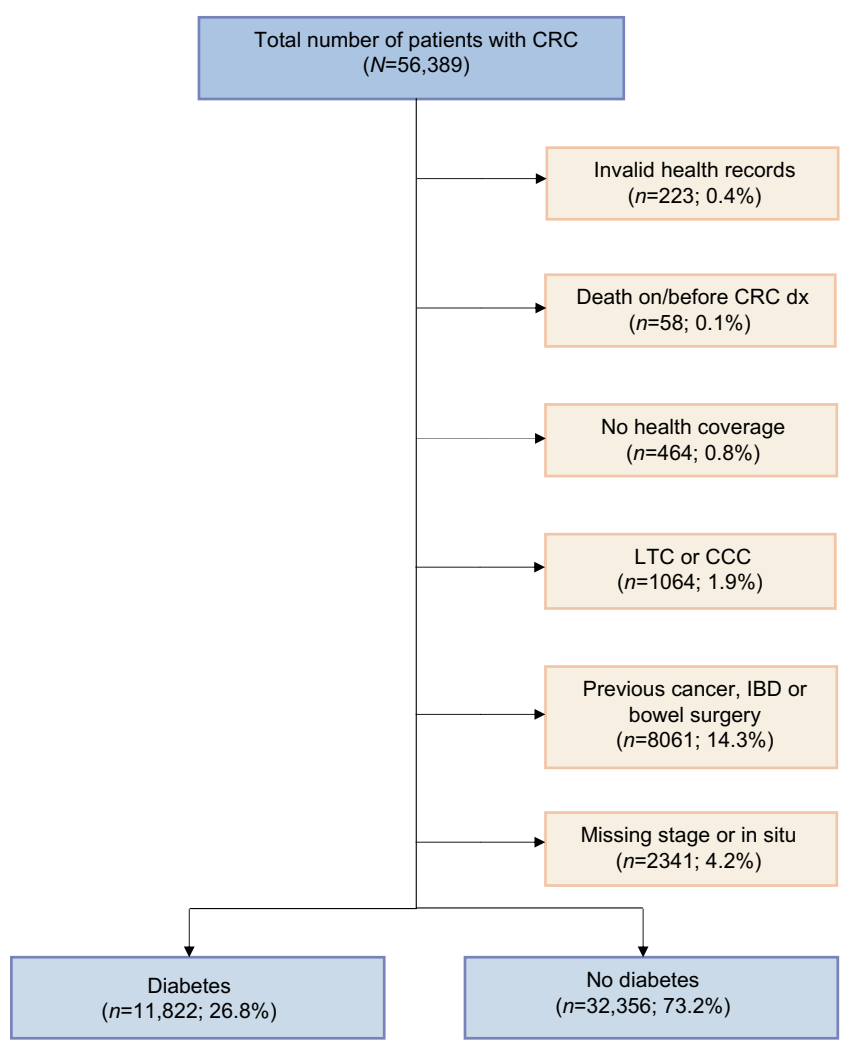

Fig. 1 Number of people with CRC and with and without diabetes after applying exclusion criteria. CCC, complex continuing care; $\mathrm{dx}$, diagnosis; IBD, inflammatory bowel disease; LTC, long-term care 
Table 1 Baseline characteristics of study cohort

\begin{tabular}{|c|c|c|c|c|}
\hline Variable & $\begin{array}{l}\text { Total } \\
(n=44,178)\end{array}$ & $\begin{array}{l}\text { No diabetes } \\
(n=32,356)\end{array}$ & $\begin{array}{l}\text { Diabetes } \\
(n=11,822)\end{array}$ & Std diff \\
\hline Mean age $^{\mathrm{a}} \pm \mathrm{SD}$ (years) & $68.7 \pm 12.7$ & $67.6 \pm 13.3$ & $71.7 \pm 10.5$ & 0.35 \\
\hline Median age $^{\mathrm{a}}$ (IQR, years) & $70(60-79)$ & $68(58-78)$ & $73(65-80)$ & 0.32 \\
\hline$<40$ years & $797(1.8)$ & $761(2.4)$ & $36(0.3)$ & 0.18 \\
\hline 40-49 years, $n(\%)$ & $2701(6.1)$ & $2426(7.5)$ & $275(2.3)$ & 0.24 \\
\hline $50-59$ years, $n(\%)$ & $7507(17.0)$ & $6165(19.1)$ & $1342(11.4)$ & 0.22 \\
\hline 60-69 years, $n(\%)$ & $11,620(26.3)$ & $8386(25.9)$ & $3235(27.4)$ & 0.03 \\
\hline $70-79$ years, $n(\%)$ & $12,255(27.7)$ & $8148(25.2)$ & $4107(34.7)$ & 0.21 \\
\hline $80+$ years, $n(\%)$ & $9298(21.0)$ & $6470(20.0)$ & $2828(23.9)$ & 0.09 \\
\hline Age $\geq 50$ years $^{\mathrm{a}}, n(\%)$ & $40,680(92.1)$ & $29,169(90.1)$ & $11,511(97.3)$ & 0.30 \\
\hline Male sex, $n(\%)$ & $24,613(55.7)$ & $17,420(53.8)$ & $7193(60.8)$ & 0.14 \\
\hline Rural residence, $n(\%)$ & $6489(14.7)$ & $4757(14.7)$ & $1732(14.7)$ & 0 \\
\hline \multicolumn{5}{|l|}{ Income quintile, $n(\%)$} \\
\hline 1 (lowest) & $8683(19.7)$ & $6061(18.7)$ & $2622(22.2)$ & 0.09 \\
\hline 2 & $9124(20.7)$ & $6515(20.1)$ & $2609(22.1)$ & 0.05 \\
\hline 3 & $8854(20.0)$ & $6510(20.1)$ & $2344(19.8)$ & 0.01 \\
\hline 4 & $8753(19.8)$ & $6526(20.2)$ & $2227(18.8)$ & 0.03 \\
\hline 5 (highest) & $8612(19.5)$ & $6644(20.5)$ & $1968(16.6)$ & 0.10 \\
\hline $\begin{array}{l}\text { Any PC visit in } 2 \text { years prior } \\
\text { to CRC diagnosis, } n(\%)\end{array}$ & $42,924(97.2)$ & $31,236(96.5)$ & $11,688(98.9)$ & 0.16 \\
\hline Up-to-date CRC screening, $n(\%)$ & $16,010(36.2)$ & $11,352(35.1)$ & $4658(39.4)$ & 0.09 \\
\hline FOBT & $10,695(24.2)$ & $7666(23.7)$ & $3029(25.6)$ & 0.04 \\
\hline Sigmoidoscopy & $5030(11.4)$ & $3480(10.8)$ & $1550(13.1)$ & 0.07 \\
\hline Colonoscopy & $6719(15.2)$ & $4632(14.3)$ & $2087(17.7)$ & 0.09 \\
\hline \multicolumn{5}{|c|}{ History of comorbidity 5 years before CRC diagnosis, $n(\%)$} \\
\hline Stroke & $336(0.8)$ & $179(0.6)$ & $157(1.3)$ & 0.08 \\
\hline MI & $1174(2.7)$ & $614(1.9)$ & $560(4.7)$ & 0.16 \\
\hline $\mathrm{CHF}$ & $974(2.2)$ & $440(1.4)$ & $534(4.5)$ & 0.19 \\
\hline \multicolumn{5}{|l|}{ Diabetes duration in years } \\
\hline Mean \pm SD & $9.1 \pm 6.3$ & & $9.1 \pm 6.3$ & \\
\hline Median (IQR) & $8(4-14)$ & & $8(4-14)$ & \\
\hline Incident diabetes, $n(\%)$ & $1781(4.0)$ & & $1781(15.1)$ & \\
\hline
\end{tabular}

${ }^{\text {a }}$ Age at CRC diagnosis

Significance denoted by standard difference equal to or greater than 0.1

PC, primary care; Std diff, standard difference stage breast cancer [25] compared with women without diabetes. Evidence regarding $\mathrm{CRC}$ cancer screening rates among people with diabetes is more conflicting [20, 24, 26, 27]. It is estimated that up to $14 \%$ of individuals with CRC have comorbid diabetes, up to $40 \%$ of whom have both diabetes and cardiovascular disease [28]. This underscores the need for a better understanding of how diabetes affects CRC and nonCRC mortality risk to guide appropriate interventions.

In light of this knowledge gap, we sought to determine whether people with diabetes who develop CRC are more likely to present with a later stage at diagnosis than those without diabetes, and the overall impact of diabetes on allcause and CRC-specific mortality (herein also referred to as cancer-specific mortality) after accounting for differences in stage at diagnosis and other potential confounders.

\section{Methods}

Data sources and setting This study used population-based, linked health administrative databases from Ontario, Canada, which include the health records of over 13 million residents covered by the universal healthcare insurance programme. We used the Cancer Care Ontario (CCO) and Ontario Cancer Registry (OCR) datasets to identify individuals with $\mathrm{CRC}$, determine CRC stage at diagnosis and capture cancer- 
Fig. 2 Percentage of people with and without diabetes presenting with each stage of colorectal cancer

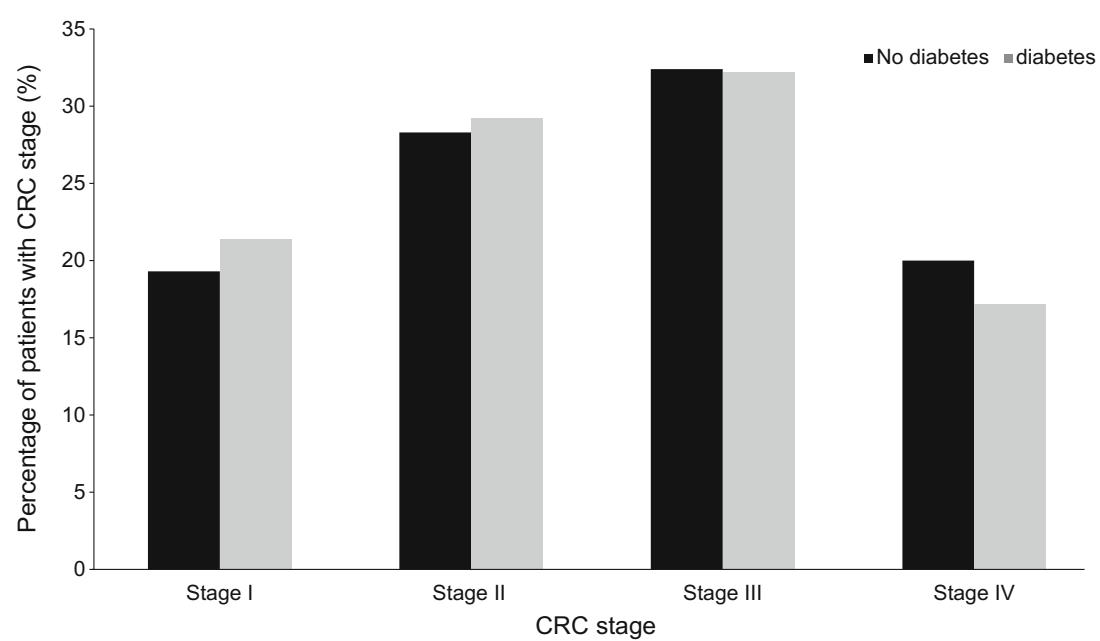

specific deaths. These are robust databases with high capture rates of cancer; for example, the OCR captures $99 \%$ of cancer cases in Ontario [29]. Non-cancer deaths were captured by the Registered Persons Database (RPDB) that is enriched with death registry records. Health services use was determined using data from the Canadian Institute for Health Information Discharge Abstract Database/Same-Day Surgery database (CIHI-DAD/SDS) and the Ontario Health Insurance Plan (OHIP) physician billing claims database. The validated Ontario Diabetes Database (ODD) was used to identify individuals with diagnosed types 1 and 2 diabetes based on physician claims and hospital discharge abstracts with a positive predictive value of $80 \%$ [30]. These datasets were linked, anonymised, encoded and analysed at ICES. This study was approved by the Research Ethics Board of Sunnybrook Hospital (Toronto, ON, Canada).

Study design and population This retrospective cohort study included all Ontario men and women aged 18 years and older diagnosed with CRC between 1 January 2007 and 31 December 2015, for whom data on cancer stage at diagnosis was available. This study was limited to Ontario residents who had health insurance coverage for at least 10 years prior to the date of CRC diagnosis to capture prior CRC screening history. Individuals with CRC stage 0 ('in situ') and those with inflammatory bowel disease, previous bowel resection, previous cancer and residing in long-term or complex continuing care were excluded, as CRC detection patterns and cancer-specific mortality may differ in these patients.

Exposure and covariates The main exposure was pre-existing diabetes at the time of CRC diagnosis. Diabetes was then further characterised as either prevalent diabetes (diabetes duration of at least 2 years) or incident diabetes (diabetes duration of less than 2 years) to isolate the effect of chronic (prevalent) from newly diagnosed (incident) diabetes on CRC stage and cancer-specific mortality.

The following covariates were recorded at baseline: age at CRC diagnosis, sex, median neighbourhood income quintile, urban vs rural residence, number of primary care visits in the 2 years prior to $\mathrm{CRC}$ diagnosis, comorbidities (including stroke, myocardial infarction [MI], congestive heart failure $[\mathrm{CHF}]$ ) and CRC screening history. Screening history was categorised as up-to-date or not prior to the CRC diagnosis
Table 2 Logistic regression comparing likelihood of laterstage (stage III or IV) vs earlystage (stage I or II) CRC at diagnosis between diabetes (all, incident, prevalent) and no diabetes, adjusted and unadjusted analysis

\begin{tabular}{|c|c|c|c|c|c|c|}
\hline \multirow[b]{2}{*}{ Diabetes } & \multicolumn{2}{|l|}{ Unadjusted } & \multicolumn{2}{|l|}{ Adjusted 1} & \multicolumn{2}{|l|}{ Adjusted 2} \\
\hline & OR $(95 \% \mathrm{CI})$ & $p$ value & OR $(95 \% \mathrm{CI})$ & $p$ value & OR $(95 \% \mathrm{CI})$ & $p$ value \\
\hline All & $0.89(0.85,0.93)$ & $<0.0001$ & $0.97(0.93,1.01)$ & 0.1596 & $0.98(0.94,1.02)$ & 0.2911 \\
\hline Incident & $0.91(0.83,1)$ & 0.0458 & $0.96(0.87,1.05)$ & 0.3553 & $0.96(0.87,1.06)$ & 0.3984 \\
\hline Prevalent & $0.89(0.85,0.93)$ & $<0.0001$ & $0.97(0.93,1.02)$ & 0.2234 & $0.98(0.93,1.03)$ & 0.3912 \\
\hline
\end{tabular}

Incident diabetes, duration $\leq 2$ years; prevalent diabetes, duration $>2$ years

Adjusted 1: adjusted for year of diagnosis, age at diagnosis, sex, income quintile, number of primary care visits in the 2 years prior to CRC diagnosis, history of $\mathrm{MI}$ and $\mathrm{CHF}$

Adjusted 2: adjusted for year of diagnosis, age at diagnosis, sex, income quintile, number of primary care visits in the 2 years prior to CRC diagnosis, history of MI and $\mathrm{CHF}$, excluding patients $<50$ years of age 
Table 3 Hazard ratios comparing all-cause and CRC-specific mortality between diabetes (all, incident, prevalent) and no diabetes

\begin{tabular}{|c|c|c|c|c|c|c|}
\hline \multirow[b]{2}{*}{ Diabetes } & \multicolumn{2}{|l|}{ Unadjusted } & \multicolumn{2}{|l|}{ Model 1} & \multicolumn{2}{|l|}{ Model 2} \\
\hline & HR $(95 \% \mathrm{CI})$ & $p$ value & $\operatorname{HR}(95 \% \mathrm{CI})$ & $p$ value & $\operatorname{HR}(95 \% \mathrm{CI})$ & $p$ value \\
\hline \multicolumn{7}{|c|}{ All-cause mortality } \\
\hline All & $1.21(1.17,1.26)$ & $<0.0001$ & $1.08(1.04,1.12)$ & $<0.0001$ & $1.12(1.08,1.16)$ & $<0.0001$ \\
\hline Incident & $1.05(0.97,1.14)$ & 0.185 & $1.01(0.93,1.09)$ & 0.8422 & $1.02(0.94,1.1)$ & 0.7152 \\
\hline Prevalent & $1.24(1.2,1.29)$ & $<0.0001$ & $1.09(1.05,1.14)$ & $<0.0001$ & $1.14(1.09,1.18)$ & $<0.0001$ \\
\hline \multicolumn{7}{|c|}{ CRC-specific mortality ${ }^{a, b}$} \\
\hline All & $1.02(0.97,1.07)$ & 0.4019 & $1.00(0.95,1.06)$ & 0.9169 & $1.04(0.99,1.10)$ & 0.1326 \\
\hline Incident & $0.97(0.87,1.08)$ & 0.5814 & $0.95(0.85,1.06)$ & 0.3562 & $0.97(0.86,1.08)$ & 0.5609 \\
\hline Prevalent & $1.03(0.98,1.09)$ & 0.2551 & $1.01(0.96,1.07)$ & 0.6367 & $1.06(1.00,1.12)$ & 0.0572 \\
\hline
\end{tabular}

Control participants are censored if they developed diabetes during the observation period. Results shown for all diabetes, incident diabetes (duration $\leq 2$ years), and prevalent diabetes (duration $>2$ years)

${ }^{\mathrm{a}}$ Taking into account competing risks of death

${ }^{\mathrm{b}}$ HRs represent the cause-specific HR

Model 1: adjusted for year of diagnosis, age at diagnosis, sex, income quintile, number of primary care visits in the 2 years prior to CRC diagnosis, history of $\mathrm{MI}$ and $\mathrm{CHF}$

Model 2: adjusted for year of diagnosis, age at diagnosis, sex, income quintile, number of primary care visits in the 2 years prior to CRC diagnosis, history of $\mathrm{MI}$ and $\mathrm{CHF}$ and CRC stage at diagnosis period (at least 6 months before CRC diagnosis) as per Canadian screening guidelines operational during our study period [31]. Individuals were considered up-to-date if they have had at least one of the following: (1) faecal occult blood test (FOBT) within 2 years to 6 months, (2) sigmoidoscopy within 5 years to 6 months or (3) colonoscopy within 10 years to 6 months prior to CRC diagnosis. Indications for endoscopy were not captured by our databases. We considered these tests performed within 6 months of CRC diagnosis as potentially diagnostic in nature and excluded them from CRC screening history. While FOBT itself is not considered a diagnostic test, a positive FOBT may trigger colonoscopy or other follow-up investigations and a subsequent diagnosis of CRC. In fact, Ontario provincial guidelines currently recommend that a positive FOBT be followed up by colonoscopy within 8 weeks [32].

Outcomes The primary outcome was CRC stage at diagnosis (categorical: stage I, II, III or IV), which was evaluated in a cross-sectional manner as a binary outcome categorised as early-stage (I and II) or later-stage (III and IV). The secondary outcomes were time to cancer-specific mortality and time to all-cause mortality. For these outcomes, patients were followed from CRC diagnosis until death. Patients alive at the end of the study period were censored at that time. Patients without diabetes were censored if they developed diabetes during the study period.

Statistical analysis The distributions of baseline characteristics among our study cohort were explored, and differences in the distributions between individuals with and without diabetes were compared using standardised differences. For the primary outcome, we performed a cross-sectional analysis using multivariable logistic regression to compare the odds of having a later-stage vs early-stage of CRC at diagnosis between patients with and without diabetes. Models were adjusted for covariates with greater than $10 \%$ standardised difference between diabetes and no diabetes groups as well as year of CRC diagnosis. These covariates included age at diagnosis, sex, income quintile, healthcare utilisation (contact with primary care), and comorbidities including MI and CHF. We adjusted for year of CRC diagnosis as this is a known confounder between prevalent diabetes and mortality risk. We also tested for interactions between sex and diabetes, and for screening history and diabetes to determine whether the effect of diabetes on CRC stage was modified by cancer screening.

To examine all-cause mortality, we implemented multivariable Cox proportional hazards regression. The main exposure in the model was the presence or absence of diabetes at CRC diagnosis and adjustment was made for the above covariates (Model 1), and additionally for CRC stage at diagnosis (Model 2). CRC-specific mortality was examined using multivariable cause-specific hazards regression, where non-CRC deaths were considered a competing risk.

For all analyses, we conducted a sensitivity analysis excluding patients under age 50 years as they are ineligible for routine CRC screening under OHIP and thus may have different detection patterns. We also repeated all analyses stratified by sex and tumour location (proximal and distal CRC). All analyses were performed using SAS 9.4, SAS Institute Inc., Cary, NC, USA. Statistical significance was defined as a $p$ value of $<0.05$. 
Fig. 3 (a) Cumulative incidence of all-cause mortality for individuals with and without diabetes; $p<0.0001$. (b) Cumulative incidence of CRCspecific and non-CRC mortality rates over time for individuals with and without diabetes (competing-risk analysis). The CRC-specific mortality curves overlap for individuals with and without diabetes a

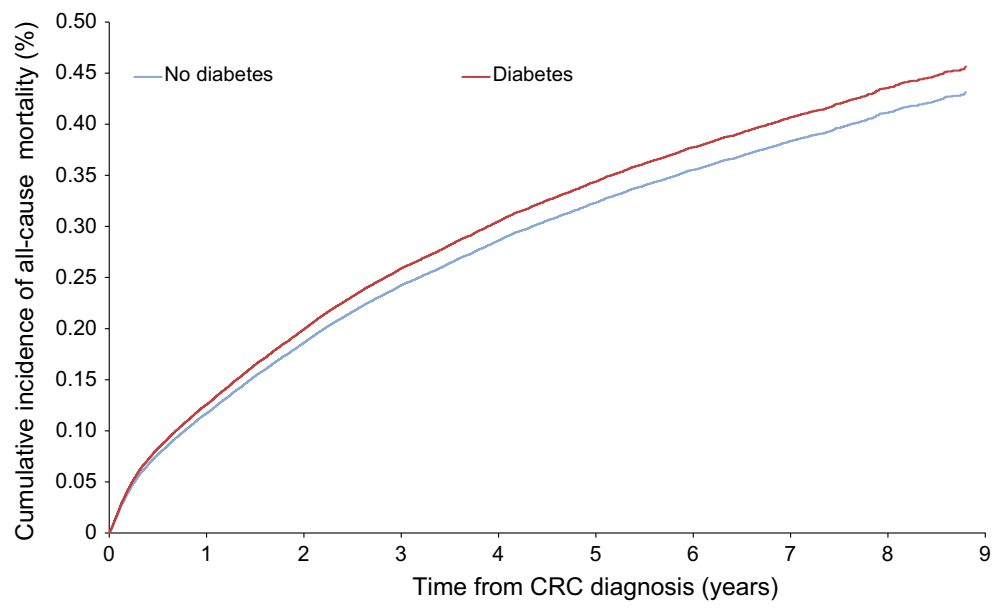

b

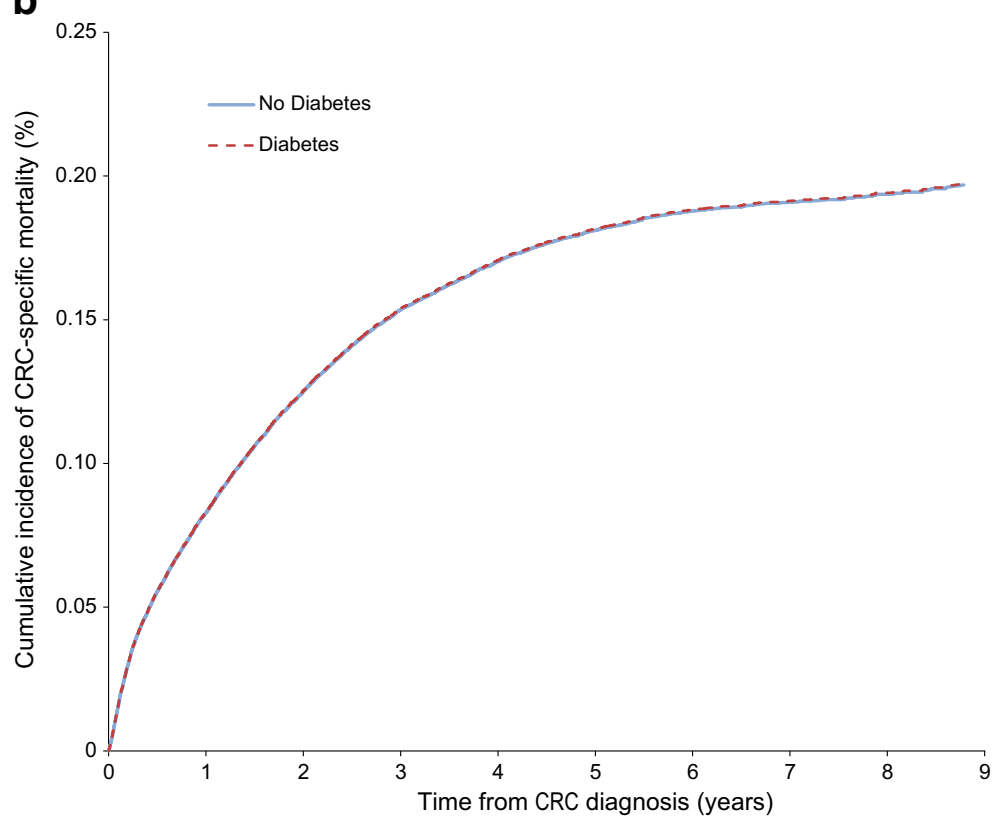

\section{Results}

Baseline characteristics We identified 56,389 individuals who were diagnosed with CRC between 2007 and 2015. After removing the 12,211 patients $(22 \%)$ who met exclusion criteria, 44,178 individuals (78\%) were included in the final analysis (Fig. 1).

As indicated by Table 1, 11,822 (26.7\%) individuals in the cohort had diabetes at baseline, and 24,613 of the cohort $(55.7 \%)$ were male. Individuals with diabetes were significantly older than those without diabetes at CRC diagnosis (median [IQR] 73 [65-80] vs 68 [58-78] years). Individuals with diabetes were more likely to visit a primary care physician 2 years prior to CRC diagnosis, and $38.6 \%$ of eligible cohort members had an up-to-date CRC screening history at 6 months before CRC diagnosis. CRC screening rates were comparable between the two groups. People with diabetes were more likely to have a history of cardiovascular disease than those without diabetes.

CRC stage at diagnosis Of the 44,178 patients who were diagnosed with CRC, 8763 (19.8\%), 12,611 (28.5\%), 14,298 (32.4\%) and $8506(19.3 \%)$ presented with stage I, II, III and IV, respectively (Fig. 2). Of the 11,822 patients with diabetes, $5973(50.5 \%)$ presented with early-stage (I or II) CRC and $5849(49.5 \%)$ presented with later-stage CRC (III or IV). Of the 32,356 patients without diabetes, 15,401 (47.6\%) presented with early-stage and $16,955(52.4 \%)$ presented with laterstage CRC.

Individuals with diabetes were more likely to present with early-stage CRC compared with individuals without diabetes (OR 0.89, 95\% CI 0.85, 0.93, $p<0.0001$ ). Given the standardised difference for CRC screening was only 0.09 $(<10 \%)$, we did not adjust for this in our main analyses. 
However, because CRC screening has an important role in $\mathrm{CRC}$ stage at diagnosis, alongside the other relevant covariates, we additionally adjusted for CRC screening in our analysis of CRC stage at diagnoses and found that the difference was no longer statistically significant (adjusted OR 0.97, 95\% CI $0.93,1.01$ ). Findings were similar for incident and prevalent diabetes, and when individuals under age 50 years were excluded (Table 2). We found no difference with respect to the likelihood of presenting with higher stage CRC at the time of diagnosis between men and women and between distal and proximal CRC (electronic supplementary material [ESM] Table 1). There were no significant interactions between diabetes and sex or diabetes and CRC screening history.

CRC-specific and all-cause mortality There were 14,785 deaths over a median (IQR) follow-up of $2.63(0.97-5.10)$ years since CRC diagnosis in the study cohort. Of those, there were 6921 (46.8\%) non-CRC deaths and 7864 (53.2\%) CRCspecific deaths. Estimated all-cause mortality was $45.4 \%$ in people with diabetes and $42.9 \%$ in people without diabetes. Estimated CRC-specific mortality was in $19.7 \%$ individuals with diabetes and $19.6 \%$ in individuals without diabetes by the end of the study period.

Individuals with diabetes had higher all-cause mortality compared with patients without diabetes, even after adjusting for relevant covariates (Model 1: adjusted HR 1.08, 95\% CI $1.04,1.12, p<0.001$ ) and additionally adjusting for CRC stage at diagnosis (Model 2: adjusted HR 1.12, 95\% CI 1.08, 1.16, $p<0.001$ ) (Table 3). This association was only significant for individuals with prevalent diabetes (greater than 2 years duration) at the time of CRC diagnosis; no effect was seen for incident diabetes (Table 3). There was no significant association between overall diabetes and CRC-specific mortality (Model 1: adjusted HR 1.0, 95\% CI 0.95, 1.06, $p=0.92$; Model 2: adjusted HR 1.04, 95\% CI 0.99, 1.1, $p=0.13$ ). Findings were similar after excluding individuals less than 50 years old (data not shown) and when we stratified analyses by sex and tumour location (ESM Table 2). However, there was a modest increase in CRC-specific mortality among patients with prevalent diabetes after adjusting for relevant covariates and excluding patients younger than 50 years of age (adjusted HR $1.06,95 \%$ CI $1.0-1.12, p=0.04)$. There were no significant interactions between diabetes and sex or diabetes and CRC screening history. Mortality risk curves are shown in Fig. 3.

\section{Discussion}

In this large population-based study, we have shown that individuals with diabetes were not more likely to present a higher stage of CRC at diagnosis compared with those without diabetes. Not surprisingly, individuals with diabetes were more likely to have comorbidities at baseline, and had higher rates of overall mortality risk compared with individuals without diabetes. However, after accounting for relevant covariates, CRCspecific mortality was similar between individuals with and without diabetes.

The effect of diabetes on CRC mortality is complex. First, there may be a biological basis for more aggressive tumour types in individuals with diabetes with poor metabolic control. Hyperglycaemia has been shown to increase vascular endothelial growth factor (VEGF) signalling, which may lead to more advanced cancers and decreased survival [33]. Second, diabetes may affect cancer treatment choices for the same stage of CRC. A Dutch group reported that clinicians may choose less aggressive CRC treatments for people with diabetes [34]. However, this finding has not been replicated in all studies. A large populationbased US study found no difference with respect to rates of CRCrelated surgeries or chemotherapy, but did show a slightly decreased rate of radiotherapy in individuals with CRC and diabetes [35].

Overall, our findings are similar to those of previously published reports. While a single-centre Taiwanese study found higher rates of CRC-specific death among individuals with diabetes [13], several other large studies have not confirmed the same elevated risk $[14,35,36]$. Interestingly, a recent population-based US study showed that in older adults (age $>67$ years), there was a modestly increased CRCspecific mortality rate only among individuals with diabetic complications, suggesting that chronicity of metabolic dysfunction and associated comorbidities may be important prognostic factors in CRC [37]. Lega et al [38] previously showed that in individuals with diabetes and breast cancer, cancer-specific mortality risk was increased only among those with longer diabetes duration and cardiovascular disease. Our data show that there is a greater CRC-specific mortality risk among individuals with prevalent diabetes compared with incident diabetes, although this did not meet statistical significance $(p=0.057)$. However, this relationship becomes statistically significant when we include only those eligible for routine CRC screening. Taken together, these findings suggest that it may be the chronicity of metabolic dysregulation rather than diabetes itself that promotes higher cancer-specific mortality risk in individuals with diabetes. Methodological variations may also contribute to the current lack of consensus in the literature regarding the impact of diabetes on CRCspecific mortality. Luo et al [35] suggested that previous studies may have over-estimated the risk of CRC-specific death by censoring individuals who experienced competing events.

In contrast, all-cause mortality has been consistently found to be higher among people with CRC and concomitant diabetes $[15,16,35,39]$. A large meta-analysis by Barone et al found that long-term all-cause mortality was increased in CRC patients with pre-existing diabetes, with a pooled hazard ratio of 1.32 $(1.24,1.41)$ [12]. Similarly, a more recent US study showed that, in older adults, pre-existing diabetes increased overall mortality 
risk among individuals with CRC (HR 1.2, 95\% CI 1.17, 1.23) [35]. The discrepancy between CRC-specific and all-cause mortality has been largely attributed to increased risk of cardiovascular deaths among individuals with diabetes [35, 40]. Overall, our findings argue against a gap in CRC-specific management for individuals with diabetes and suggest that a greater focus on non-cancer care, including diabetes management and optimisation of cardiovascular health, is needed to improve clinical outcomes after cancer.

Previous studies reported conflicting data with respect to cancer screening among individuals with diabetes. While studies suggest that individuals with diabetes may have inadequate breast and cervical cancer screening, possibly contributing to worse clinical outcomes, the studies on the uptake of CRC screening tests are more conflicting [20, 41-43]. Furthermore, the evidence on CRC screening in individuals with diabetes has significant limitations resulting from low methodological quality and many of the studies being conducted in the USA, where access to screening may be less equitable. A recent meta-analysis of diabetes and cancer screening studies found that while diabetes was not associated with lower CRC screening overall, CRC screening was significantly lower among women with diabetes compared with those without [24]. In our study, CRC screening rates were comparable between both men and women with diabetes. This finding may be due to greater opportunities for CRC screening in individuals with diabetes through more frequent clinical interactions, particularly in our universally funded healthcare system. On the other hand, an earlier Canadian study showed that despite having more primary care visits, fewer women with diabetes received breast cancer screening [25]. One possibility is that during routine diabetes follow-up, gastrointestinal (GI) symptoms are more likely to be noted than breast symptoms. For example, GI symptoms may be discussed in the context of side effects of oral hypoglycaemic agent, or with comorbidities such as coeliac disease or gastroparesis. Any abnormal symptoms may then trigger further investigations and GI referrals, which may include testing for CRC.

Evidence for the association between diabetes and CRC stage at diagnosis has been mixed in the literature $[44,45]$. Our data show that stage at CRC diagnosis is similar between individuals with and without diabetes, after adjusting for relevant covariates. These results are corroborated by another large population-based study, which showed no significant difference with respect to CRC stage at diagnosis or tumour grade when comparing individuals with and without diabetes [35]. It has been suggested that early-stage disease is more likely to be detected in those with diabetes because of increased contact with healthcare providers, which may bias towards a null effect of diabetes by mitigating its potential adverse effects on cancer growth [46]. Indeed, we found no difference in stage at CRC diagnosis between individuals with and without diabetes. Our results suggest that in the context of a well-established universal healthcare system, potential negative effects of diabetes on CRC outcomes may be offset by the greater opportunities for early disease detection that are afforded by access to regular diabetes care.

Our study has several strengths. First, the use of validated and comprehensive registries, with high rates of disease capture, involving data tied to physician financial reimbursement allowed us to accurately identify individuals with diabetes and CRC, which strengthens our conclusions. Second, use of large, population-based datasets allowed us to minimise potential confounders that may be problematic in studies involving single centres or subsets of the general population, and increases the generalisability of our data. Third, the universal nature of the Ontario healthcare system allowed for equitable provision of and opportunity for CRC screening, thus removing barrier to care as a potential confounding factor. Finally, we used a competing-risk statistical model, which allowed us to account for multiple, mutually exclusive events, to generate accurate survival estimates.

Several weaknesses should also be mentioned. There are inherent biases and unmeasured confounders in a retrospective study, which may affect our conclusions. For example, we could not examine the effect of $\mathrm{HbA}_{1 \mathrm{c}}$, degree of diabetes control or diabetes medication use on CRC-related outcomes. Higher $\mathrm{HbA}_{1 \mathrm{c}}$ has been shown to have negative prognostic value in CRC [47]. The impact of diabetes treatment, such as metformin, on risk of CRC and mortality risk outcomes has been of interest in the literature, without definitive conclusions so far [27, 47, 48]. These clinical questions would be important to address in future studies. In addition, we did not capture BMI, ethnicity, smoking status, alcohol use or family history, which are all important risk factors for CRC. While we did not directly adjust for diabetes duration, we did subcategorise patients with diabetes into incident (diabetes duration $\leq 2$ years) and prevalent (diabetes duration $>2$ years) diabetes in order to isolate the effect of chronic metabolic dysfunction on CRC-specific and overall mortality risk. We do recognise that we may have missed some undiagnosed cases of diabetes at the time of CRC diagnosis, but these are likely to be small as CRC diagnosis is most often made on final pathology, after surgical or endoscopic resection, which would have provided opportunities for diabetes detection. In addition, we did not measure all relevant comorbidities, such as renal failure, which may have CRC treatment implications and therefore affect mortality rates. While beyond the scope of this study, incorporation of CRC treatment as a predictor of mortality risk in individuals with diabetes would be helpful and could be investigated in the future.

In summary, in this large population-based study of individuals with CRC, we have demonstrated that the presence of diabetes did not significantly affect the stage at CRC diagnosis or cancer-specific mortality. However, individuals with diabetes had higher all-cause mortality after a CRC diagnosis compared with individuals without diabetes. These results underscore the 
need for a greater focus on non-cancer care to reduce mortality risk among individuals with $\mathrm{CRC}$ and diabetes.

Acknowledgements We thank ServiceOntario for use of Office of the Registrar General (ORG) information on deaths. The views expressed herein do not necessarily reflect those of ORG or the Ministry of Government Services.

Data availability The dataset from this study is held securely in coded form at ICES. While data sharing agreements prohibit ICES from making the dataset publicly available, access may be granted to those who meet pre-specified criteria for confidential access, available at www.ices.on.ca/ DAS. The full dataset creation plan and underlying analytic code are available from the authors upon request, understanding that the programs may rely upon coding templates or macros that are unique to ICES.

Funding LLL is supported by Diabetes Investigator Award from Diabetes Canada. This study was supported by the Ontario Cancer Institute for Research and Cancer Care Ontario. This study was also supported by ICES, which is funded by an annual grant from the Ontario Ministry of Health and Long-Term Care (MOHLTC). The opinions, results and conclusions reported in this paper are those of the authors and are independent from the funding sources. No endorsement by ICES or the Ontario MOHLTC is intended or should be inferred. Parts of this material are based on data and/or information compiled and provided by CIHI. However, the analyses, conclusions, opinions and statements expressed in the material are those of the author(s), and not necessarily those of CIHI. Parts of this material are based on data and information provided by Cancer Care Ontario (CCO). The opinions, results, view, and conclusions reported in this paper are those of the authors and do not necessarily reflect those of CCO. No endorsement by $\mathrm{CCO}$ is intended or should be inferred.

Duality of interest The authors declare that there is no duality of interest associated with this manuscript.

Contribution statement All authors fulfil the following criteria: (1) substantial contributions to conception and design, acquisition of data, or analysis and interpretation of data; (2) drafting the article or revising it critically for important intellectual content; and (3) final approval of the version to be published. JQ, LLL, DB and RS drafted the manuscript. LLL is the guarantor of this work.

\section{References}

1. Harding JL, Shaw JE, Peeters A, Cartensen B, Magliano DJ (2015) Cancer risk among people with type 1 and type 2 diabetes: disentangling true associations, detection bias, and reverse causation. Diabetes Care 38(2):264-270. https://doi.org/10.2337/dc141996

2. Vigneri P, Frasca F, Sciacca L, Pandini G, Vigneri R (2009) Diabetes and cancer. Endocr Relat Cancer 16(4):1103-1123. https://doi.org/10.1677/ERC-09-0087

3. Hense HW, Kajuter H, Wellmann J, Batzler WU (2011) Cancer incidence in type 2 diabetes patients - first results from a feasibility study of the D2C cohort. Diabetol Metab Syndr 3(1):15. https://doi. org/10.1186/1758-5996-3-15

4. Noto H, Tsujimoto T, Sasazuki T, Noda M (2011) Significantly increased risk of cancer in patients with diabetes mellitus: a systematic review and meta-analysis. Endocr Pract 17(4):616628. https://doi.org/10.4158/EP10357.RA

5. Sun G, Kashyap SR (2011) Cancer risk in type 2 diabetes mellitus: metabolic links and therapeutic considerations. J Nutr Metab 2011: 708183

6. Peeters P, Bazelier MT, Leufkens H, de Vries F, De Bruin ML (2015) The risk of colorectal cancer in patients with type 2 diabetes: associations with treatment stage and obesity. Diabetes Care 38(3): 495-502. https://doi.org/10.2337/dc14-1175

7. Tsilidis KK, Kasimis JC, Lopez DS, Ntzani EE, Ioannidis JP (2015) Type 2 diabetes and cancer: umbrella review of meta-analyses of observational studies. BMJ 350:g7607

8. Bray F, Ferlay J, Soerjomataram I, Siegel RL, Torre LA, Jemal A (2018) Global cancer statistics 2018: GLOBOCAN estimates of incidence and mortality worldwide for 36 cancers in 185 countries. CA Cancer J Clin 68(6):394-424. https://doi.org/10.3322/caac. 21492

9. Lega IC, Wilton AS, Austin PC, Fischer HD, Johnson JA, Lipscombe LL (2016) The temporal relationship between diabetes and cancer: a population-based study. Cancer 122(17):2731-2738. https://doi.org/10.1002/cncr.30095

10. De Jong RGPJ, Burden AM, de Kort S et al (2017) Impact of detection bias on the risk of gastrointestinal cancer and its subsites in type 2 diabetes mellitus. Eur J Cancer 79:61-71. https://doi.org/ 10.1016/j.ejca.2017.03.039

11. Pollak M (2012) The insulin and insulin-like growth factor receptor family in neoplasia: an update. Nat Rev Cancer 12(3):159-169. https://doi.org/10.1038/nrc3215

12. Barone BB, Yeh HC, Snyder CF et al (2008) Long-term all-cause mortality in cancer patients with preexisting diabetes mellitus: a systematic review and meta-analysis. JAMA 300(23):2754-2764. https://doi.org/10.1001/jama.2008.824

13. Huang YC, Lin JK, Chen WS et al (2011) Diabetes mellitus negatively impacts survival of patients with colon cancer, particularly in stage II disease. J Cancer Res Clin Oncol 137(2):211-220. https:// doi.org/10.1007/s00432-010-0879-7

14. Van de Poll-Franse LV, Haak HR, Coebergh JW, Janssen-Heijnen ML, Lemmens VE (2012) Disease-specific mortality among stage I-III colorectal cancer patients with diabetes: a large populationbased analysis. Diabetologia 55(8):2163-2172. https://doi.org/10. 1007/s00125-012-2555-8

15. Stein KB, Snyder CF, Barone BB et al (2010) Colorectal cancer outcomes, recurrence, and complications in persons with and without diabetes mellitus: a systematic review and meta-analysis. Dig Dis Sci 55(7):1839-1851. https://doi.org/10.1007/s10620-0090944-8

16. Jullumstro E, Kollind M, Lydersen S, Edna TH (2009) Diabetes mellitus and outcomes of colorectal cancer. Acta Oncol 48(3): 361-367. https://doi.org/10.1080/02841860802637765

17. Voutsadakis IA (2017) Obesity and diabetes as prognostic factors in patients with colorectal cancer. Diabetes Metab Syndr 11(Suppl 1): S109-S114

18. Tarantino I, Achermann P, Guller U et al (2013) Relative survival is an adequate estimate of cancer-specific survival: baseline mortalityadjusted 10-year survival of 771 rectal cancer patients. Ann Surg Oncol 20(12):3877-3884. https://doi.org/10.1245/s10434-0133173-5

19. American Joint Committee on Cancer (2010) Small intestine. In: Edge SB, Byrd DR, Compton CC, Fritz AG, Greene FL, Trotti A (eds) AJCC Cancer Staging Manual, 7th edn. Springer, New York, p 143

20. McBean AM, Yu X (2007) The underuse of screening services among elderly women with diabetes. Diabetes Care 30(6):14661472. https://doi.org/10.2337/dc06-2233 
21. Lipscombe LL, Hux JE, Booth GL (2005) Reduced screening mammography among women with diabetes. Arch Intern Med 165(18):2090-2095. https://doi.org/10.1001/archinte.165.18.2090

22. Constantinou P, Dray-Spira R, Menvielle G (2016) Cervical and breast cancer screening participation for women with chronic conditions in France: results from a national health survey. BMC Cancer 16:255

23. Chan W, Yun L, Austin PC et al (2014) Impact of socio-economic status on breast cancer screening in women with diabetes: a population-based study. Diabet Med 31(7):806-812. https://doi. org/10.1111/dme.12422

24. Bhatia D, Lega IC, Wu W, Lipscombe LL (2020) Breast, cervical and colorectal cancer screening in adults with diabetes: a systematic review and meta-analysis. Diabetologia 63(1):34-48. https://doi. org/10.1007/s00125-019-04995-7

25. Lipscombe LL, Fischer HD, Austin PC et al (2015) The association between diabetes and breast cancer stage at diagnosis: a populationbased study. Breast Cancer Res Treat 150(3):613-620. https://doi. org/10.1007/s10549-015-3323-5

26. Greiner KA, Daley CM, Epp A et al (2014) Implementation intentions and colorectal screening. Am J Prev Med 47(6):703-714. https://doi.org/10.1016/j.amepre.2014.08.005

27. Liu BY, O’Malley J, Mori M et al (2014) The association of type and number of chronic diseases with breast, cervical, and colorectal cancer screening. J Am Board Fam Med 27(5):669-681. https://doi. org/10.3122/jabfm.2014.05.140005

28. Cuthbert C, Hemmelgarn BR, Xu Y, Cheung WY (2018) The effect of comorbidities on outcomes in colorectal cancer survivors: a population-based cohort study. J Cancer Surviv 12(6):733-743. https://doi.org/10.1007/s11764-018-0710-z

29. Robles SC, Marrett LD, Clarke EA, Risch HA (1988) An application of capture-recapture methods to the estimation of completeness of cancer registration. J Clin Epidemiol 41(5):495-501. https:// doi.org/10.1016/0895-4356(88)90052-2

30. Hux JE, Ivis F, Flintoft V, Bica A (2002) Diabetes in Ontario: determination of prevalence and incidence using a validated administrative data algorithm. Diabetes Care 25(3):512-516. https://doi. org/10.2337/diacare.25.3.512

31. Canadian Task Force on Preventative Health Care (2001) Colorectal cancer screening: recommendation statement from the Canadian Task Force on Preventive Health Care. CMAJ 165(2): 206-208

32. Cancer Care Ontario. Abnormal fecal occult blood test result frequently asked questions. Available from https://www. cancercareontario.ca/en/types-of-cancer/colorectal/screening/ abnormal-fobt-result-faq. Accessed 1 Nov 2019

33. Yang IP, Tsai HL, Huang CW et al (2016) High blood sugar levels significantly impact the prognosis of colorectal cancer patients through down-regulation of microRNA-16 by targeting $M y b$ and VEGFR2. Oncotarget 7(14):18837-18850. https://doi.org/10. 18632/oncotarget.7719

34. van de Poll-Franse LV, Houterman S, Janssen-Heijnen ML, Dercksen MW, Coebergh JW, Haak HR (2007) Less aggressive treatment and worse overall survival in cancer patients with diabetes: a large population based analysis. J Cancer 120(9):1986-1992

35. Luo J, Lin HC, He K, Hendryx M (2014) Diabetes and prognosis in older persons with colorectal cancer. Br J Cancer 110(7):18471854. https://doi.org/10.1038/bjc.2014.68
36. Bella F, Minicozzi P, Giacomin A et al (2013) Impact of diabetes on overall and cancer-specific mortality in colorectal cancer patients. J Cancer Res Clin Oncol 139(8):1303-1310. https://doi.org/10.1007/ s00432-013-1439-8

37. El Brahimi S, Smith ML, Pinheiro PS (2019) Role of pre-existing type 2 diabetes in colorectal cancer survival among older Americans: a SEER-Medicare population-based study 20022011. Int J Color Dis 34(8):1467-1475. https://doi.org/10.1007/ s00384-019-03345-8

38. Lega IC, Austin PC, Fischer HD et al (2018) The impact of diabetes on breast cancer treatments and outcomes: a population-based study. Diabetes Care 41(4):755-761. https://doi.org/10.2337/dc172012

39. Zhu B, Wu XM, Wu B, Pei D, Zhang L, Wei L (2017) The relationship between diabetes and colorectal cancer prognosis: a metaanalysis based on the cohort studies. PLoS One 12(4):e0176068. https://doi.org/10.1371/journal.pone.0176068

40. Worndl E, Fung K, Fischer HD, Austin PC, Krzyzanowska MK, Lipscombe LL (2018) Preventable diabetic complications after a cancer diagnosis in patients with diabetes: a population-based cohort study. JNCI Cancer Spectr 2(1):pky008

41. Fontana SA, Baumann LC, Helberg C, Love RR (1997) The delivery of preventive services in primary care practices according to chronic disease status. Am J Public Health 87(7):1190-1196. https://doi.org/10.2105/ajph.87.7.1190

42. Bell RA, Shelton BJ, Paskett ED (2001) Colorectal cancer screening in North Carolina: associations with diabetes mellitus and demographic and health characteristics. Prev Med 32(2):163-167. https://doi.org/10.1006/pmed.2000.0785

43. Zhao G, Ford ES, Ahluwalia IB, Li C, Mokdad AH (2009) Prevalence and trends of receipt of cancer screenings among US women with diagnosed diabetes. J Gen Intern Med 24(2):270-275. https://doi.org/10.1007/s11606-008-0858-8

44. Fazio L, Cotterchio M, Manno M, McLaughlin J, Gallinger S (2005) Association between colonic screening, subject characteristics, and stage of colorectal cancer. Am J Gastroenterol 100(11): 2531-2539. https://doi.org/10.1111/j.1572-0241.2005.00319.x

45. De Marco MF, Janssen-Heijnen ML, van der Heijden LH, Coebergh JW (2000) Comorbidity and colorectal cancer according to subsite and stage: a population-based study. Eur J Cancer 36(1): 95-99. https://doi.org/10.1016/s0959-8049(99)00221-x

46. Johnson JA, Bowker SL, Richardson K, Marra CA (2011) Timevarying incidence of cancer after the onset of type 2 diabetes: evidence of potential detection bias. Diabetologia 54(9):22632271. https://doi.org/10.1007/s00125-011-2242-1

47. Ferroni P, Formica V, Della-Morte D et al (2016) Prognostic value of glycated hemoglobin in colorectal cancer. Wolrd J Gastroenterol 22(45):9984-9993

48. Paulus JK, William CD, Crossor FI, Kelley MJ, Martell RE (2016) Metformin, diabetes, and survival among U.S. veterans with colorectal cancer. Cancer Epidemiol Biomark Prev 25(10):1418-1425

Publisher's note Springer Nature remains neutral with regard to jurisdictional claims in published maps and institutional affiliations. 